\title{
Super-stretchable borophene
}

This content has been downloaded from IOPscience. Please scroll down to see the full text.

\section{EPL 11636001}

(http://iopscience.iop.org/0295-5075/116/3/36001)

View the table of contents for this issue, or go to the journal homepage for more

Download details:

IP Address: 159.226.199.8

This content was downloaded on 25/04/2017 at 09:52

Please note that terms and conditions apply.

You may also be interested in:

Strain effects on borophene: ideal strength, negative Possion's ratio and phonon instability Haifeng Wang, Qingfang Li, Yan Gao et al.

Effects of strain on mechanical and electronic properties of borophene

Li Shao, Yan Li, Qingxin Yuan et al.

Mechanical properties of borophene films: a reactive molecular dynamics investigation Minh Quy Le, Bohayra Mortazavi and Timon Rabczuk

The effect of transverse loading on the ideal tensile strength of face-centered-cubic materials Duc Tam Ho, Soon-Dong Park, Soon-Yong Kwon et al.

Anisotropic mechanical and optical response and negative Poisson's ratio in Mo2C nanomembranes revealed by first-principles simulations

Bohayra Mortazavi, Masoud Shahrokhi, Meysam Makaremi et al.

The defect-induced fracture behaviors of hexagonal boron-nitride monolayer nanosheets under uniaxial tension

Xiong Qi-lin, Li Zhen-huan and Tian Xiao-geng

The mechanical exfoliation mechanism of black phosphorus to phosphorene: A first-principles study

Yunsheng Mu and M. S. Si

Unexpected buckled structures and tunable electronic properties in arsenic nanosheets: insights from first-principles calculations

Yanli Wang and Yi Ding 


\title{
Super-stretchable borophene
}

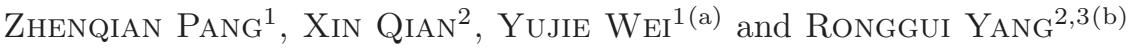 \\ 1 The State Key Laboratory of Nonlinear Mechanics (LNM), Institute of Mechanics, Chinese Academy of Sciences \\ Beijing, 100190, PRC \\ 2 Department of Mechanical Engineering, University of Colorado - Boulder, CO 80309, USA \\ 3 Materials Science and Engineering Program, University of Colorado - Boulder, CO 80309, USA
}

received 25 August 2016; accepted in final form 23 November 2016

published online 19 December 2016

PACS 62.20.de - Elastic moduli

PACS 62.20.dj-Poisson's ratio

\begin{abstract}
Recent success in the synthesis of the two-dimensional borophene on silver substrates has attracted strong interest in exploring its extraordinary properties for potential technological applications. The single-layer borophene has a buckled structure with atomic ridges. By using the first-principles density functional theory calculations, we show that the two-dimensional borophene is highly stretchable with strong anisotropy The strain-to-failure in the direction along the atomic ridges is nearly twice as large as that across the atomic ridges. The straining-induced flattening and the subsequent stretch of the flat borophene are accounted for the large strain-to-failure for tension along the atomic ridges. We also investigated the mechanics of monolayer borophene under biaxial tension and we found that the biaxial tension increases the strength across the atomic ridges but decreases the failure strain along the atomic ridges. Furthermore, when the bilayer borophene is stretched along the cross-plane direction, the strength and failure strain of the bilalyer borophene are much higher than those of the bilayer graphene due to the very strong inter-layer chemical bonding.
\end{abstract}

Copyright (C) EPLA, 2016

Since the successful synthesis of the single-layer hexagonal boron-nitride (h-BN) [1,2], the community of two-dimensional materials has been curious about the possibility of making monolayer and few-layer borophene polymorphs [3-5] Boron resides on the left side of carbon in the periodic table while having valence orbitals similar to carbon, making it possible to form new twodimensional (2-D) structures to extend the family of 2-D materials [6-8]. However, boron cannot form a stable 2-D hexagonal honeycomb structure like graphene [9-11]. For example, the 2-D $\mathrm{B}_{36}$ cluster with a central hexagonal hole has been synthesized [9]. More flexible 2-D boron clusters, such as the $\mathrm{B}_{35}$ cluster with a double-hexagonal vacancy [12], and $B_{30}$ clusters [13] have also been predicted by first-principles density functional theory (DFT) calculations. As for 2-D boron sheets (borophene), the first-principles calculations predict the structure to be stable with hexagonal vacancies [7] on metal surfaces [5]. It was until very recently that different borophene structures have been synthesized on silver substrates, with either hexagonal hole [14] or atomic ridges recently observed

\footnotetext{
(a) E-mail: yujie_wei@lnm.imech.ac.cn (corresponding author)

(b) E-mail: ronggui.yang@colorado.edu (corresponding author)
}

using a scanning tunnelling microscope [15]. It is of great interest to examine the unconventional physical properties of borophene that might be originated from its 2-D nature. For example, 2-D borophene shows metallic behaviour [15] even though the three-dimensional boron is nonmetallic and semiconductive. Because the borophene is both metallic [16] and atomically thin, it holds great promises for potential applications ranging from electronics [17] to photovoltaics. Recently, the mechanical property and the negative Poisson ratio of monolayer borophene have also attracted much interest [18], the mechanical properties of the bilayer borophene, however, have not been studied yet. In this work, we perform a systematic investigation on the mechanical properties of the monolayer borophene synthesized by Mannix et al. [15] as well as its corresponding bilayer counterpart [19], including Young's modulus, the Poisson ratio, the strength and the mechanical stability under strain.

First-principles density functional theory (DFT) calculations for the mechanical properties of borophene were performed using the Vienna $A b$ initio Simulation Package (VASP) code [20,21]. The projector augmentedwave (PAW) pseudopotentials $[22,23]$ and the generalized 

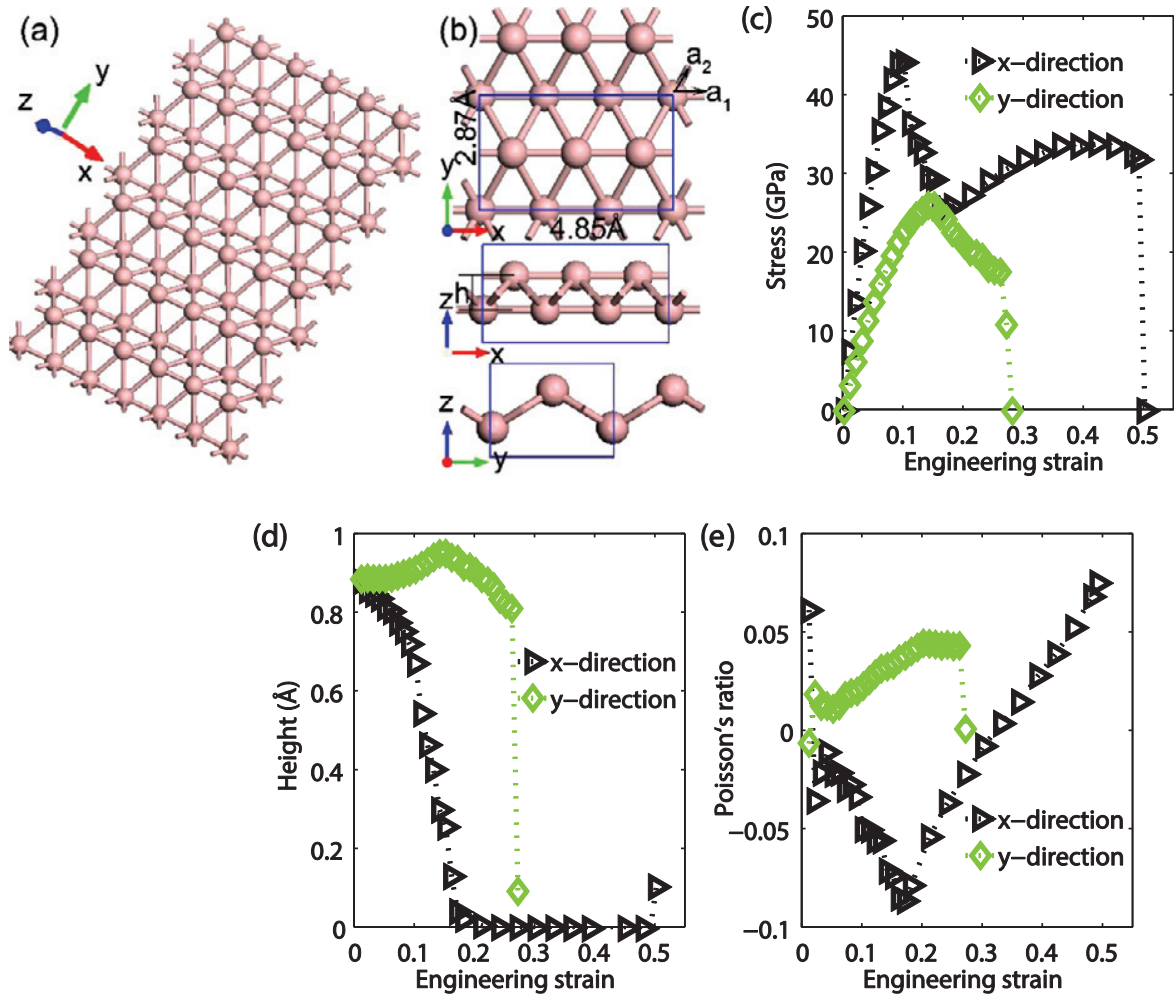

Fig. 1: (Color online) Mechanical behaviour of the monolayer borophene under uniaxial tension in the $x-y$ plane: (a) atomic structure of the monolayer borophene. (b) The monolayer borophene viewed from the top ( $x-y$ plane) and the side $(x-z$ plane, and $y$ - $z$ plane). (c) The stress-strain curves for uniaxial loading along the $x$-direction and the $y$-direction. (d) Evolution of the layer height ( $h$ in the $z$-direction) under straining in $x$ - or $y$-directions. (e) Poisson's ratio vs. mechanical strains along the $x$ and $y$-directions.

gradient approximation (GGA) of the Perdew-BurkeErnzerhof (PBE) functional $[24,25]$ are used. A planewave basis set with a kinetic-energy cut-off of $400 \mathrm{eV}$ and a Monkhorst-Pack [26] $k$-point mesh of $31 \times 31 \times 1$ are used for the static electronic structure calculations. Periodic boundary conditions are applied in the two in-plane directions for all the calculations conducted here. All structures are relaxed using a conjugate gradient algorithm until the atomic forces are converged to $0.01 \mathrm{eV} / \AA$. To eliminate the interactions between periodic images of boron atoms, a vacuum space of $20 \AA$ was used both in monolayer and bilayer borophene calculations

As illustrated in fig. 1(a) and (b) the monolayer borophene has a buckled structure with parallel atomic ridges along the $x$-axis. For convenience, the twodimensional primitive cell is specified using two lattice vectors $\boldsymbol{a}_{1}$ and $\boldsymbol{a}_{2}$ as shown in fig. 1(b). Mechanical loading is applied along two different directions in the $x-y$ plane, I) in the direction $\boldsymbol{a}=(1,0)$ along the $x$-axis in the Cartesian coordinate, and II) in the direction $\boldsymbol{a}=(1,2)$ along the $y$-axis in the Cartesian coordinates. Included in fig. 1(b) are the equilibrium lattice constants (conventional unit cell indicated by blue box) obtained by DFT calculations, $4.85 \AA$ by $2.87 \AA$, which agree well with the experimental measurement of $5.10 \AA$ by $2.9 \AA$ in the $x$ - and $y$-direction, respectively [15]. To obtain the in-plane stress of monolayer borophene with the physical unit comparable to three-dimensional stress in pascals $(\mathrm{Pa})$, a nominal thickness of $4.8 \AA$ is assumed here, which equals the inter-layer distance obtained by relaxing the AA-stacked borophene monolayers. We first show the engineering stress-strain curves for the samples subjected to uniaxial tension along the $x$ - and $y$-directions (fig. 1(c)). Similar to the mechanical properties of h-BN [27], we find that the strength and the failure strain in the monolayer borophene are strongly anisotropic. When the tension is applied in the $x$-direction, the monolayer borophene shows both higher strength and failure strain than those in the $y$-direction. There exist two stress peaks in the stressstrain curves along the $x$-direction compared with that in the $y$-direction: the first peak occurs at a strain of $10 \%$ and the second one appears at a strain of $45 \%$. The change of atomic layer height is also anisotropic when strains are imposed in the $x$ - and $y$-directions shown in fig. $1(\mathrm{~d})$. When strains are imposed in the $x$-direction, it is observed that the layer height decreases with increasing strain and the structure eventually flattens out with $h=0$ at $18 \%$ strain along the $x$-axis. Interestingly, under the tension along the $x$-direction, the stress increases again when the sample is fully flattened (at 18\% strain); the material fails at a high strain of $50 \%$. In contrast, the sample fails at a strain of $27 \%$ under the tension in the $y$-direction. 

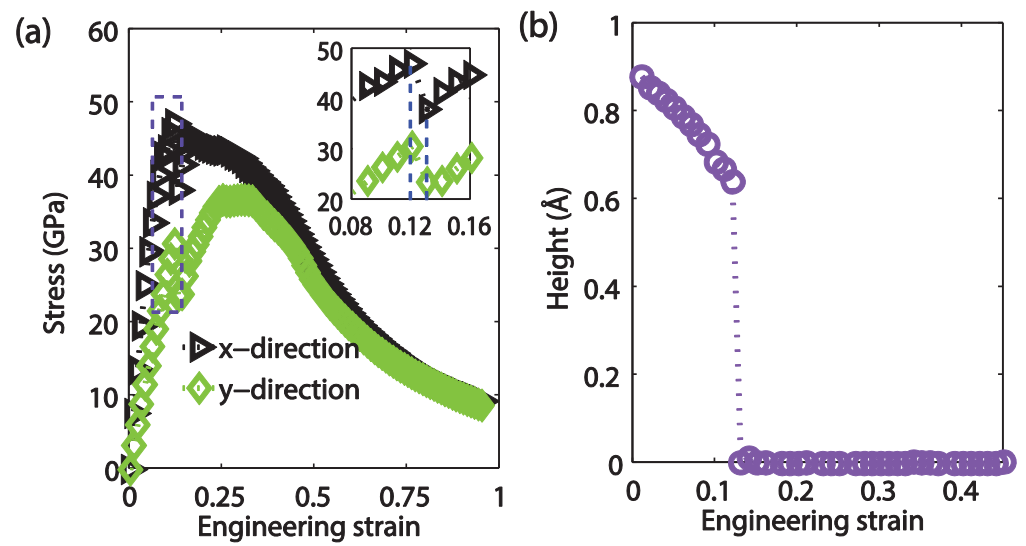

Fig. 2: (Color online) The mechanical response of the monolayer borophene under biaxial tension. (a) The stress as a function of strain in both directions. (b) The evolution of the height as a function of the biaxial strain.
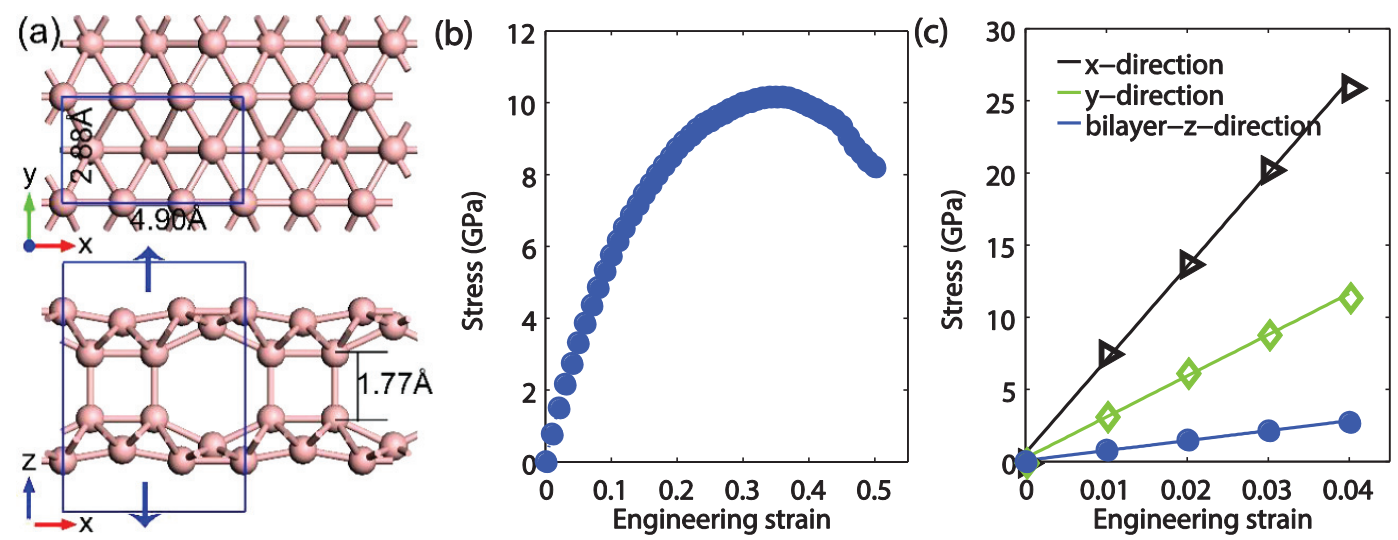

Fig. 3: (Color online) Anistropic elastic properties of the bilayer borophene. (a) The bilayer borophene viewed from the top (x-y plane) and the side ( $x-z$ plane). The covalent bonding length is $1.77 \AA$ in the bilayer borophene. When strain is imposed along the $z$-axis across the atomic layer, the interlayer distance is changed and the vacuum layer distance of $20 \AA$ is kept constant. (b) The stress as a function of the strain in the $z$-direction. (c) The stress-strain curves under small elastic strain for tensile loading along three directions.

Interestingly, the layer height is first increased when small strains are applied in the $y$-direction. This can be understood by calculating Poisson's ratio $\nu$ as a function of the tensile strain as shown in fig. 1(e). Poisson's ratio is defined as $\nu=-\frac{L_{L}-L 0_{L}}{L 0_{T}-L 0_{T}} \frac{L 0_{L}}{L 0_{L}}$, where $L 0_{T}$ and $L 0_{L}$ are the initial lengths of the unit cell in the loading direction and in the direction perpendicular to the loading within the atomic layer, respectively, while $L_{T}$ and $L_{L}$ are the corresponding ones after tension. Our calculation shows that Poisson's ratio is also strongly anisotropic. When the tension is applied in the $y$-direction, Poisson's ratio is positive and the monolayer borophene would contract in the $x$-axis. Such deformation tends to reduce the angle formed by the atoms at the bottom and the peak of the ridges (fig. 1(b), middle), which increases the atomic layer thickness. Therefore, when small strains are applied along the $y$-axis, the height of the borophene ridges is increased first. The negative Poisson ratio is observed when the tension is applied along the $x$-direction. Our calculation results agree with the observation in ref. [15].
Similar to the single-layer black phosphorus [28] and penta-graphene [29], such negative Poisson's ratio originates from the buckled atomic structures.

In addition to the uniaxial stress-strain response, we further explore the stress-strain response of the monolayer borophene under the biaxial tension as shown in fig. 2(a). A unique mechanical response is observed during the biaxial stretching where a sudden stress drop occurs at a strain of $12 \%$ in the two stress-strain response curves. The sudden stress drop in the bi-axial response curves is due to the flattening of the monolayer borophene from the buckled structure to the planar structure (fig. 2(b)), which is similar to the case in which uniaxial stretch in the $y$-direction is applied. However, the buckled-to-planar structure transition only occurs gradually in the uniaxial stretch case when the $x$-direction is stretched (fig. 1(d)). Compared with the uniaxial tension (fig. 1(c)), we observe that stretching along the $x$-direction greatly enhances the subsequent strength in the $y$-direction. After the sudden stress drop, the stress increases again and the second 
peak appears. We relax the structures for strains within $18 \% \pm 8 \%$ by assigning a small perturbation to the lattice constants. Structures with strain larger than $17 \%$ will evolve to a disordered state. This indicates that the maximum biaxial strain is $17 \%$; beyond this point, the structure is not stable from the total-energy point of view.

We further studied the mechanics of the bilayer borophene. Figure 3(a) shows the structure and lattice constants of a bilayer borophene. The lattice constants are $4.90 \AA$ and $2.88 \AA$ in the $x$ - and $y$-directions, respectively. Each layer has a similar but distorted structure compared to that of the mononlayer borophene in fig. 1(a). Different from other layered materials [30] where the weak van der Waals forces dominate interlayer coupling, the two monolayers in the bilayer borophene crystal are bonded together with very strong and short chemical bonds (bonding length of $1.7 \AA$ ) This unique feature suggests that the bilayer borophene should have a considerable high inter-layer separation strength. By applying mechanical stretch in the inter-layer direction (fig. 3(b)), the peak stress is observed when applying a strain of $38 \%$. The covalent bonding length connecting two monolayers increased from $1.77 \AA$ (unstrained) to $2.44 \AA$ (at $38 \%$ strain). When a strain of $50 \%$ is applied, the bilayer borophene structure fails at a bonding length of $2.66 \AA$. The separation strength extracted for a bilayer borophene crystal is as high as $10 \mathrm{GPa}$, which is considerably greater than that of the graphite where interlayers are bonded by van der Waals force (about $3 \mathrm{GPa}$ ) [31]. In fig. 3(c), we show the detailed stress-strain relationship for the two in-plane directions and the out-of-plane direction in the bilayer borophene, under small strains. Young's modulus is $646.6 \mathrm{GPa}$ in the $x$-direction and $285.2 \mathrm{GPa}$ in the $y$-direction. The modulus of separating bilayer borophene along the $z$-direction $(68.8 \mathrm{GPa}$ ) is the smallest.

In summary, we investigated several critical mechanical properties of the monolayer borophene and the bilayer borophene by using first-principles DFT calculations. We find that the failure behavior, Young's modulus and Poisson's ratio of both the monolayer and bilayer borophene are highly anisotropic: Poisson's ratio is negative when tension is along the atomic ridge direction. For the bilayer borophene, the calculated strength and the failure strain are much higher than that of the bilayer graphene due to the strong inter-layer chemical bonding.

$$
* * *
$$

YW acknowledges the support from the National Natural Science Foundation of China (NSFC) (Grant No. 11425211). RY acknowledges the support from National Science Foundation (Grant No. 1512776). Calculations are performed at the Supercomputing Center of CAS and the Janus supercomputer supported by National Science Foundation (Grant No. 0821794) and University of Colorado Boulder.

\section{REFERENCES}

[1] Novoselov K. S., Jiang D., Schedin F., Booth T. J., Khotkevich V. V., Morozov S. V. and Geim A. K., Proc. Nat. Acad. Sci. U.S.A., 102 (2005) 10451.

[2] Chopra N. G., Luyken R. J., Cherrey K., Crespi V. H., Cohen M. L., Louie S. G. and Zettl A., Science, 269 (1995) 966.

[3] Lau K. C. and Pandey R., J. Phys. Chem. C, 111 (2007) 2906.

[4] Penev E. S., Bhowmick S., Sadrzadeh A. and YAKobson B. I., Nano Lett., 12 (2012) 2441.

[5] Liu Y., Penev E. S. and Yakobson B. I., Angew. Chem., Int. Ed. Engl., 52 (2013) 3156.

[6] Zhai H.-J., Zhao Y.-F., Li W.-L., Chen Q., Bai H., Hu H.-S., Piazza Z. A., Tian W.-J., Lu H.-G., Wu Y.-B., Mu Y.-W., Wei G.-F., Liu Z.-P., Li J., Li S.-D. and WANG L.-S., Nat. Chem., 6 (2014) 727.

[7] Yang X., Ding Y. and Ni J., Phys. Rev. B, 77 (2008) 041402.

[8] Tang H. and Ismail-Beigi S., Phys. Rev. Lett., 99 (2007) 115501.

[9] Piazza Z. A., Hu H.-S., Li W.-L., Zhao Y.-F., Li J. and Wang L.-S., Nat. Commun., 5 (2014) 3113.

[10] Lau K. C. and Pandey R., J. Phys. Chem. C, 111 (2007) 2906.

[11] Evans M. H., Joannopoulos J. D. and Pantelides S. T., Phys. Rev. B, 72 (2005) 045434.

[12] Li W.-L., Chen Q., Tian W.-J., Bai H., Zhao Y.-F., Hu H.-S., Li J., Zhai H.-J., Li S.-D. and WANG L.-S., J. Am. Chem. Soc., 136 (2014) 12257.

[13] Li W.-L., Zhao Y.-F., Hu H.-S., Li J. and Wang L.-S., Angew. Chem., Int. Ed. Engl., 126 (2014) 5646.

[14] Feng B., Zhang J., Zhong Q., Li W., Li S., Li H., Cheng P., Meng S., Chen L. and Wu K., Nat. Chem., 8 (2016) 563.

[15] Mannix A. J., Zhou X.-F., Kiraly B., Wood J. D., Alducin D., Myers B. D., Liu X., Fisher B. L., Santiago U. and Guest J. R., Science, 350 (2015) 1513.

[16] Liu Y., Dong Y.-J., Tang Z., Wang X.-F., Wang L., Hou T., Lin H. and Li Y., J. Mater. Chem. C, 4 (2016) 6380.

[17] Peng B., Zhang H., Shao H., Xu Y., Zhang R. and Zhu H., J. Mater. Chem. C, 4 (3592) 2016.

[18] Wang Haifeng, Li Qingfang, Gao Yan, Miao F., Zhou Xiang-Feng and Wan X. G., New J. Phys., 18 (2016) 073016.

[19] Zhou X.-F., Dong X., Oganov A. R., Zhu Q., Tian Y. and Wang H.-T., Phys. Rev. Lett., 112 (2014) 085502 .

[20] Kresse G. and Furthmüller J., Comput. Mater. Sci., 6 (1996) 15.

[21] Kresse G. and Furthmüller J., Phys. Rev. B, 54 (1996) 11169.

[22] Blöchl P. E., Phys. Rev. B, 50 (1994) 17953.

[23] Kresse G. and Joubert D., Phys. Rev. B, 59 (1999) 1758.

[24] Perdew J. P., Burke K. and Ernzerhof M., Phys. Rev. Lett., 77 (1996) 3865. 
[25] Perdew J. P., Burke K. and Ernzerhof M., Phys. Rev. Lett., 78 (1997) 1396.

[26] Monkhorst H. J. and PACK J. D., Phys. Rev. B, 13 (1976) 5188.

[27] Wu J., Wang B., Wei Y., Yang R. and Dresselhaus M., arXiv preprint, arXiv:1301.2104 (2013).

[28] Jiang J.-W. and Park H. S., Nat. Commun., 5 (2014) 4727.
[29] Zhang S., Zhou J., Wang Q., Chen X., Kawazoe Y. and Jena P., Proc. Nat. Acad. Sci. U.S.A., 112 (2015) 2372.

[30] Rydberg H., Dion M., Jacobson N., Schröder E., Hyldgandd P., Simak S. I., Langreth D. C. and Lundqvist B. I., Phys. Rev. Lett., 91 (2003) 126402.

[31] Spanu L., Sorella S. and Galli G., Phys. Rev. Lett., 103 (2009) 196401. 